\title{
Terminology in morphological anomalies of 1 crasentax the cerebellum does matter
}

\author{
Andrea Poretti ${ }^{1,2^{*}}$ and Eugen Boltshauser ${ }^{2}$
}

\begin{abstract}
Neuroimaging plays a key role in the diagnostic work-up of morphological abnormalities of the cerebellum. Diagnostic criteria for numerous morphological anomalies of the cerebellum are based on neuroimaging findings. Various morphological patterns have been described on neuroimaging including cerebellar hypoplasia, cerebellar agenesis, pontocerebellar hypoplasia, cerebellar dysplasia, cerebellar dysmorphia, and cerebellar atrophy. These patterns have specific differential diagnoses. The familiarity with the diagnostic criteria is mandatory for a correct diagnosis and a targeted work-up to avoid unnecessary investigations. A correct diagnosis is essential for early therapy, prognosis, and counseling of the affected children and their family.
\end{abstract}

Keywords: Cerebellum, Neuroimaging, Cerebellar atrophy, Cerebellar hypoplasia, Cerebellar dysplasia, Cerebellar dysmorphia

\section{Background}

Progress in neuroimaging techniques and genetic analysis has led to a significant improvement in definition of morphological abnormalities of the cerebellum. New classifications and checklists of morphological abnormalities of the cerebellum have been proposed [1-3].

Neuroimaging plays a key role in the diagnostic work-up of morphological abnormalities of the cerebellum $[4,5]$. Diagnostic criteria for numerous morphological anomalies of the cerebellum are based on neuroimaging findings. Various morphological patterns have been described on neuroimaging including cerebellar hypoplasia, cerebellar agenesis, pontocerebellar hypoplasia, cerebellar dysplasia, cerebellar dysmorphia, and cerebellar atrophy. These patterns have specific differential diagnoses. The familiarity with the diagnostic criteria is mandatory for a correct diagnosis and a targeted work-up to avoid unnecessary investigations. An accurate diagnosis of these complex abnormalities is important for: 1) early institution of the correct therapy, 2) prediction of the prognosis,

\footnotetext{
* Correspondence: aporett1@jhmi.edu

'Section of Pediatric Neuroradiology, Division of Pediatric Radiology, Russell H. Morgan Department of Radiology and Radiological Science, The Johns Hopkins University School of Medicine, 1800 Orleans Street, Baltimore, MD 21287, USA

${ }^{2}$ Department of Pediatric Neurology, University Children's Hospital, Zurich,
} Switzerland

and 3) counseling of the family including inheritance pattern and risk of recurrence.

Sometimes in the daily clinical work as well as in the scientific literature, the terms mentioned above are used interchangeably. This leads to confusion and may result in misdiagnosis. Semantics does matter in medical and scientific communications [6]. Precision in the use of language, whether verbal or written, is a reflection of precision in scientific thought and patient care.

Here, we will 1) differentiate between cerebellar malformations and cerebellar disruptions and 2) define and discuss various morphological cerebellar patterns including cerebellar agenesis, cerebellar hypoplasia, pontocerebellar hypoplasia, cerebellar dysplasia, cerebellar dysmorphia, and cerebellar atrophy.

\section{Cerebellar malformations versus cerebellar disruptions}

The diagnosis of congenital cerebellar anomalies should include the differentiation between inherited (developmental) and acquired (disruptive) abnormalities. A malformation is defined as a congenital morphologic anomaly of a single organ or body part due to cellular and molecular pathways involved in organogenesis; the molecules in these pathways can be altered by gene mutations, teratogens, or combined effects [7]. A disruption is defined as a congenital morphologic anomaly due to the breakdown of a body structure that had a normal developmental 
potential [7]. Disruptions may be caused by e.g. prenatal infection, hemorrhage, or ischemia and commonly involve the cerebellum [2]. Disruptions are acquired lesions with very low recurrence risk. However, a genetic predisposition to disruptive lesions may be present. Dominant mutations in COL4A1 lead to change of the basal membrane of capillaries resulting in microangiopathy [8]. Within the brain, the microangiopathy may lead to hemorrhage and/ or ischemia and result in a spectrum of lesions including porencephaly or unilateral cerebellar hypoplasia $[9,10]$.

\section{Cerebellar hypoplasia}

Cerebellar hypoplasia is a descriptive term implying a cerebellum with a reduced volume, but a normal shape and is stable over time (Fig. 1a-c) [11]. Cerebellar hypoplasia is a rather common finding and is associated with a highly heterogeneous group of diseases. Etiologies include prenatal infections and exposure to teratogens, chromosomal aberrations, metabolic disorders, genetic syndromes, and brain malformations. These include primary malformative and secondary disruptive lesions.

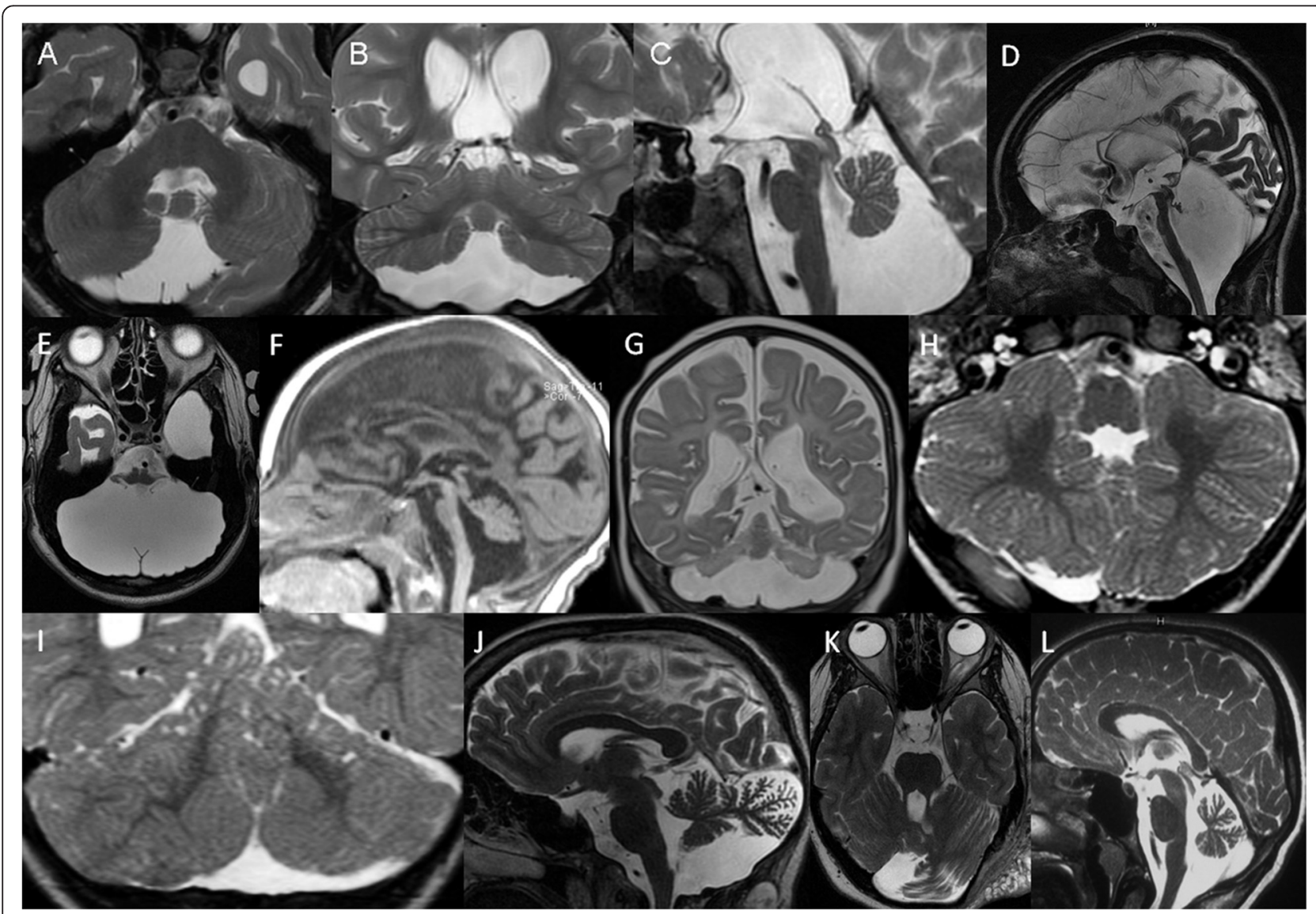

Fig. 1 a, Axial, b, Coronal, and c, Sagittal T2-weighted images of a 17-year-old male with seizures and cerebellar hypoplasia of unknown origin show reduced volume, but near normal structure of the cerebellum. In addition, a cavum Vergae and reduced periventricular bilateral white matter are noted. $\mathbf{d}$ Sagittal and e, Axial T2-weighted images of a 15-year-old girl with cerebellar agenesis reveal almost complete absence of cerebellar tissue except for a rudimentary structure projecting posterior to the inferior colliculi and lateral to the brainstem. The pons is markedly hypoplastic and the posterior fossa is enlarged (reprinted with permission from Poretti A et al., Eur J Paediatric Neurol, 2009). f Sagittal T1- and g Axial T2-weighted images of a 1-year-old child with pontocerebellar hypoplasia type 2 and TSEN54 mutations show marked hypoplasia and reduction in the size of the cerebellar hemispheres with relative preservation of the midline vermis, resulting in a characteristic "dragonfly" appearance (reprinted with permission from Bosemani T et al., Radiographics, 2015). h Axial and i, Coronal T2-weighted images of an 11-month-old child with Poretti-Boltshauser syndrome and LAMA1 mutations reveal bilateral cerebellar dysplasia as abnormal cerebellar foliation, white matter arborization, and gray-white matter junction and multiple cortical/subcortical cysts located within the cerebellar vermis (mostly anterior and superior part) and both cerebellar hemispheres (mostly posterior and superior parts) (reprinted with permission from Poretti A et al., Cerebellum, 2014). j Sagittal and $\mathbf{k}$, Axial T2-weighted images of 15-year-old patient with neurofibromatosis type 1 and cerebellar dysmorphia show enlargement of the left cerebellar hemisphere with enlarged interfolial spaces and bulky appearance of its posteromedial part, which crosses the midline. In addition, a plexiform neurofibroma is seen in the left soft tissue (reprinted with permission from Toelle SP et al., Cerebellum, 2015). I Sagittal T2-weighted image of a 5-year-old child with ataxia oculomotor apraxia type 1 disease and marked cerebellar atrophy as enlargement of the interfolial spaces 
The cerebellar involvement is heterogeneous: hypoplasia may affect the entire cerebellum (most commonly) or selectively involve the vermis alone or one/both hemispheres sparing the vermis.

Cerebellar hypoplasia may also present with enlarged cerebellar sulci (mimicking cerebellar atrophy), that is stable over time (in contrast to cerebellar atrophy). Because of the non-progressive course, we prefer the term hypoplasia to describe this imaging pattern [12-14].

Unilateral morphological anomalies of the cerebellum (e.g. unilateral cerebellar hypoplasia and unilateral cerebellar clefts) result from prenatal acquired injuries $[15,16]$. In support of this concept, second trimester or early third trimester prenatal cerebellar hemorrhages have been shown by fetal MRI [17]. In addition, some patients have associated destructive lesions such as schizencephaly.

\section{Cerebellar agenesis}

Cerebellar agenesis is defined by the near complete absence of cerebellar tissue with only remnants of the anterior vermis, flocculus, and/or middle cerebellar peduncles (Fig. 1d-e). A secondary pontine hypoplasia is typically seen. The definition of cerebellar agenesis is based on the morphologic pattern and does not suggest the pathogenesis [2]. Cerebellar agenesis may represent a malformation (e.g., mutations in PTF1A) [18] or a disruption (e.g., hemorrhage that occurs during gestation or in the perinatal period, vascular insufficiency in Chiari II malformation and cerebellar herniation, and as a sequela of prematurity) $[2,19]$.

\section{Pontocerebellar hypoplasia}

The term pontocerebellar hypoplasia is often used in a descriptive manner to imply that the volume of the cerebellum and the pons is reduced. On the other hand, it is used specifically to refer to the pontocerebellar hypoplasias conceptualized by Peter Barth as early (prenatal) onset degenerative disorder (types 1, 2, 4, 5, 6, and 7) [20]. In this sense, the term "hypoplasia" is misleading and atrophy would be more accurate. In the last years, however, diseases with a non-progressive course have been included in the heterogeneous group of pontocerebellar hypoplasias (types 3 and 8). Listing in OMIM does not reflect pathogenesis.

Pontocerebellar hypoplasias as conceptualized by Peter Barth have a peculiar neuroimaging pattern including more pronounced involvement of the cerebellar hemispheres compared to the vermis and reduction in size of the pons (Fig. 1f-g) [21]. Predominant involvement of the cerebellar hemispheres is unusual and results in a "dragonfly" appearance on coronal images: flattened cerebellar hemispheres represent "the wings", while the relatively preserved vermis represents "the body". A "dragonfly" appearance has been shown also in very low birth weight (less than $1500 \mathrm{~g}$ ) premature infants born before 32 weeks of gestation [22] and CASK mutations [23].

Reduction in size of the pons is not seen in the vast majority of diseases associated with cerebellar atrophy with postnatal onset [1]. Pontine hypoplasia however is a feature of diseases associated with prenatal loss of cerebellar tissue: 1) hereditary disorders with prenatal onset cerebellar atrophy including the group of pontocerebellar hypoplasias [21] and congenital disorder of glycosylation type 1a due to PMM2 mutations [24], and 2) acquired disorders with prenatal onset cerebellar disruptive lesions such as cerebellar agenesis [2] and vanishing cerebellum in myelomeningocele [25], and anencephaly [26]. In addition, marked reduction in size of the pons has been shown in very low birth weight (less than $1500 \mathrm{~g})$ premature infants born before 32 weeks of gestation [22, 27].

\section{Cerebellar dysplasia}

Cerebellar dysplasia is defined by abnormal cerebellar foliation, white matter arborization, and gray-white matter junction (Fig. 1h). Dysplasia may globally involve the cerebellum or affect only one cerebellar hemisphere. In addition, cerebellar dysplasia may be associated with cortical/subcortical cysts [28]. Cerebellar cysts are likely the result of disturbed cortical migration/organization and pial membrane disruption and are most likely formed from the subarachnoid spaces that were engulfed by the dysplastic cerebellar folia, particularly in the boundary between the normal and dysplastic cerebellar cortex.

Global cerebellar dysplasia has been reported in a few posterior fossa malformations including ChudleyMcCullough syndrome [29], $\alpha$-dystroglycanopathies [30], GPR56-related polymicrogyria [31], and PorettiBoltshauser syndrome due to LAMA1 mutations [32, 33]. In $\alpha$-dystroglycanopathies [30], GPR56-related polymicrogyria [31], and Poretti-Boltshauser syndrome due to LAMA1 mutations [32,33], cerebellar dysplasia is typically associated with cerebellar cysts. Diagonal folia across the vermis on axial images have been reported in tubulinopathies [34]. Dysplasia of the superior cerebellar vermis is generally seen in Joubert syndrome and can be very helpful when the other neuroimaging features (e.g. the molar tooth sign) are subtle or distorted [35]. Finally, cerebellar dysplasia may be the result of a disruptive process such as a prenatal hemorrhage. Disruptive cerebellar dysplasia may be uni- or bilateral and the affected hemispheres may be reduced in volume. In prenatal cerebellar disruptions, focal cerebellar dysplasia is typically confined to the region of the disrupted injury such as a cerebellar cleft [15]. In a few patients, disruptive cerebellar dysplasia may be associated with focal cerebellar cysts [28]. For specific diagnosis, it is important to determine the pattern of dysplasia and the presence of 
cerebellar cysts (Fig. 1i) and correlate them with clinical information [28].

Lhermitte-Duclos disease or "dysplastic cerebellar gangliocytoma" (OMIM 158350) is a rare disorder of the cerebellum that is usually included into the group of focal cerebellar dysplasia [36]. In contrast to ChudleyMcCullough syndrome, $\alpha$-dystroglycanopathies, GPR56related polymicrogyria, and Poretti-Boltshauser syndrome, in Lhermitte-Duclos disease the orientation of the cerebellar foliae is preserved, while the volume of multiple cerebellar foliae is diffusely increased [37]. This results in a characteristic neuroimaging pattern of localized lamellar hypertrophy: the inner portions of the folia are T1 hypointense and T2 hyperintense, while the outer portions are $\mathrm{T} 1$ isointense and $\mathrm{T} 2$ iso- to hypointense.

\section{Cerebellar dysmorphia}

Cerebellar dysmorphia is a term that we recently coined to refer to peculiar morphological anomalies that we saw in few patients with neurofibromatosis type 1 [38]. Cerebellar dysmorphia refers to enlargement of a cerebellar hemisphere with widening of the interfolial spaces of its posterior part, which is bulky (like an appendicular portion of additional cerebellar tissue) and crosses the midline (Fig. 1j-k).

\section{Cerebellar atrophy}

Cerebellar atrophy is a relatively common neuroimaging finding in pediatric neurology and neuroradiology. Cerebellar atrophy is defined as a cerebellum with initially normal structures, in a posterior fossa with normal size,

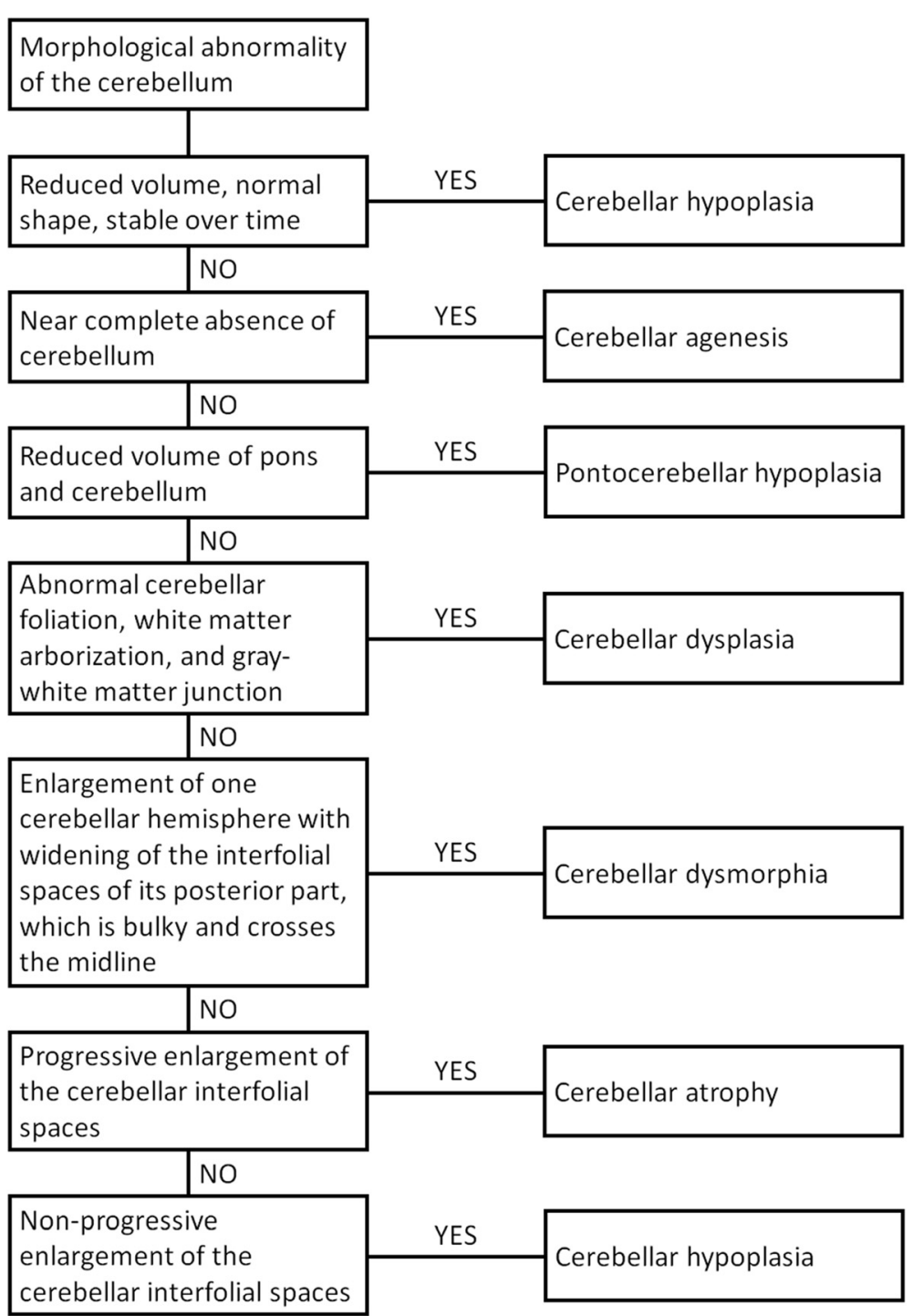

Fig. 2 Flow chart developed for reviewing MRI studies displaying morphological abnormalities of the cerebellum 
which displays enlarged fissures (interfolial spaces) in comparison to the foliae secondary to loss of tissue (Fig. 11) [1]. Cerebellar atrophy implies irreversible loss of tissue and result from an ongoing progressive disease until a final stage is reached or a single injury, e.g. an intoxication or infectious event.

Cerebellar atrophy is a non-specific neuroimaging finding and has been associated with a long list of pediatric diseases including genetic and acquired causes. We proposed a pattern recognition approach for hereditary pediatric cerebellar atrophy [1]. We differentiated between isolated ("pure") cerebellar atrophy and cerebellar atrophy associated ("plus") with additional neuroimaging findings including hypomyelination, progressive white matter abnormalities, signal change of the dentate nucleus, cerebellar cortex T2-hyperintensity, and basal ganglia involvement.

The distinction between cerebellar atrophy and cerebellar hypoplasia is not difficult in theory, but can be problematic or impossible in practice based on a single examination. In children with non-progressive cerebellar ataxia (i.e. with an obviously longstanding static situation), enlarged cerebellar sulci mimicking cerebellar atrophy CA may be seen and are stable over time (stable follow-up up to 20 years) [12-14]. In this situation, we prefer the term cerebellar hypoplasia because of the non-progressive course of clinical and neuroimaging findings and favor a malformative instead of a degenerative pathomechanism. In addition, cerebellar atrophy may be superimposed to cerebellar hypoplasia as shown in some forms of pontocerebellar hypoplasia and some children with congenital disorder of glycosylation type 1a [21, 24].

\section{Conclusion}

Neuroimaging enables the distinction between various morphological pattern of cerebellar anomalies including cerebellar agenesis, cerebellar hypoplasia, pontocerebellar hypoplasia, cerebellar dysplasia, cerebellar dysmorphia, and cerebellar atrophy (Fig. 2). For these patterns, different diagnostic criteria based on neuroimaging findings have been delineated. Familiarity with these diagnostic criteria is mandatory for a correct diagnosis and a targeted work-up to avoid unnecessary investigations. A correct diagnosis is essential for early therapy, prognosis, and counseling of the affected children and their family.

\section{Competing interests}

The authors declare that they have no competing interests.

\section{Authors' contributions}

AP and EB have been involved in drafting the manuscript and take public responsibility. $A P$ and $E B$ have given final approval of the manuscript.

Received: 5 June 2015 Accepted: 27 June 2015

Published online: 07 July 2015

\section{References}

1. Poretti A, Wolf $\mathrm{NI}$, Boltshauser E. Differential diagnosis of cerebellar atrophy in childhood. Eur J Paediatr Neurol. 2008;12(3):155-67.

2. Poretti A, Prayer D, Boltshauser E. Morphological spectrum of prenatal cerebellar disruptions. Eur J Paediatr Neurol. 2009;13(5):397-407.

3. Barkovich AJ, Millen KJ, Dobyns WB. A developmental and genetic classification for midbrain-hindbrain malformations. Brain. 2009; 132(Pt 12):3199-230.

4. Bosemani T, Orman G, Boltshauser E, Tekes A, Huisman TA, Poretti A. Congenital abnormalities of the posterior fossa. Radiographics. 2015;35(1):200-20.

5. Arora R. Imaging spectrum of cerebellar pathologies: a pictorial essay. Pol J Radiol. 2015;80:142-50.

6. Sarnat HB. Semantics do matter! Precision in scientific communication in pediatric neurology. J Child Neurol. 2007;22(11):1245-51.

7. Hennekam RC, Biesecker LG, Allanson JE, Hall JG, Opitz JM, Temple IK, et al. Elements of morphology: general terms for congenital anomalies. Am J Med Genet A. 2013;161A(11):2726-33.

8. Gould DB, Phalan FC, Breedveld GJ, van Mil SE, Smith RS, Schimenti JC, et al. Mutations in Col4a1 cause perinatal cerebral hemorrhage and porencephaly. Science. 2005;308(5725):1167-71.

9. Vermeulen RJ, Peeters-Scholte C, Van Vugt JJ, Barkhof F, Rizzu P, van der Schoor SR, et al. Fetal origin of brain damage in 2 infants with a COL4A1 mutation: fetal and neonatal MRI. Neuropediatrics. 2011;42(1):1-3.

10. Yoneda Y, Haginoya K, Kato M, Osaka H, Yokochi K, Arai H, et al. Phenotypic spectrum of COL4A1 mutations: porencephaly to schizencephaly. Ann Neurol. 2013;73(1):48-57.

11. Poretti A, Boltshauser E, Doherty D. Cerebellar hypoplasia: Differential diagnosis and diagnostic approach. Am J Med Genet C: Semin Med Genet. 2014;166(2):211-26.

12. Yapici Z, Eraksoy M. Non-progressive congenital ataxia with cerebellar hypoplasia in three families. Acta Paediatr. 2005;94(2):248-53.

13. Turkmen S, Demirhan O, Hoffmann K, Diers A, Zimmer C, Sperling K, et al. Cerebellar hypoplasia and quadrupedal locomotion in humans as a recessive trait mapping to chromosome 17p. J Med Genet. 2006;43(5):461-4.

14. Boltshauser E, Poretti A. Nonprogressive congenital ataxia. In: Boltshauser E, Schmahmann JD, editors. Cerebelalr disorders in children. London: Mac Keith Press; 2012. p. 135-9.

15. Poretti A, Leventer RJ, Cowan FM, Rutherford MA, Steinlin M, Klein A, et al. Cerebellar cleft: a form of prenatal cerebellar disruption. Neuropediatrics. 2008;39(2):106-12.

16. Poretti A, Limperopoulos C, Roulet-Perez E, Wolf NI, Rauscher C, Prayer D, et al. Outcome of severe unilateral cerebellar hypoplasia. Dev Med Child Neurol. 2010;52(8):718-24.

17. Malinger G, Zahalka N, Kidron D, Ben-Sira L, Lev D, Lerman-Sagie T. Fatal outcome following foetal cerebellar haemorrhage associated with placental thrombosis. Eur J Paediatr Neurol. 2006;10(2):93-6.

18. Sellick GS, Barker KT, Stolte-Dijkstra I, Fleischmann C, Coleman RJ, Garrett C, et al. Mutations in PTF1A cause pancreatic and cerebellar agenesis. Nat Genet. 2004;36(12):1301-5.

19. Poretti A, Risen S, Meoded A, Northington FJ, Johnston MV, Boltshauser E, et al. Cerebellar agenesis: an extreme form of cerebellar disruption in preterm neonates. J Pediatr Neuroradiol. 2013;2:163-7.

20. Namavar Y, Barth PG, Poll-The BT, Baas F. Classification, diagnosis and potential mechanisms in pontocerebellar hypoplasia. Orphanet J Rare Dis. $2011 ; 6: 50$.

21. Namavar $Y$, Barth PG, Kasher PR, van Ruissen F, Brockmann K, Bernert G, et al. Clinical, neuroradiological and genetic findings in pontocerebellar hypoplasia. Brain. 2011;134(Pt 1):143-56.

22. Messerschmidt A, Brugger PC, Boltshauser E, Zoder G, Sterniste W, Birnbacher $\mathrm{R}$, et al. Disruption of cerebellar development: potential complication of extreme prematurity. AJNR Am J Neuroradiol. 2005;26(7):1659-67.

23. Burglen L, Chantot-Bastaraud S, Garel C, Milh M, Touraine R, Zanni G, et al. Spectrum of pontocerebellar hypoplasia in 13 girls and boys with CASK mutations: confirmation of a recognizable phenotype and first description of a male mosaic patient. Orphanet J Rare Dis. 2012;7:18.

24. Feraco P, Mirabelli-Badenier M, Severino M, Alpigiani MG, Di Rocco M, Biancheri $R$, et al. The shrunken, bright cerebellum: a characteristic MR finding in congenital disorders of glycosylation type 1a. AJNR Am J Neuroradiol. 2012;33(11):2062-7. 
25. Boltshauser E, Schneider J, Kollias S, Waibel P, Weissert M. Vanishing cerebellum in myelomeningocoele. Eur J Paediatr Neurol. 2002;6(2):109-13.

26. Poretti A, Meoded A, Ceritoglu E, Boltshauser E, Huisman TA. Postnatal in-vivo MRI findings in anencephaly. Neuropediatrics. 2010;41(6):264-6.

27. Zafeiriou DI, Ververi A, Anastasiou A, Soubasi V, Vargiami E. Pontocerebellar hypoplasia in extreme prematurity: clinical and neuroimaging findings. Pediatr Neurol. 2013;48(1):48-51.

28. Boltshauser $\mathrm{E}$, Scheer I, Huisman TA, Poretti A. Cerebellar cysts in children: a pattern recognition approach. Cerebellum. 2015;14(3):308-16.

29. Doherty D, Chudley AE, Coghlan G, Ishak GE, Innes AM, Lemire EG, et al. GPSM2 mutations cause the brain malformations and hearing loss in Chudley-McCullough syndrome. Am J Hum Genet. 2012;90(6):1088-93.

30. Clement E, Mercuri E, Godfrey C, Smith J, Robb S, Kinali M, et al. Brain involvement in muscular dystrophies with defective dystroglycan glycosylation. Ann Neurol. 2008;64(5):573-82.

31. Bahi-Buisson N, Poirier K, Boddaert N, Fallet-Bianco C, Specchio N, Bertini E, et al. GPR56-related bilateral frontoparietal polymicrogyria: further evidence for an overlap with the cobblestone complex. Brain. 2010;133(11):3194-209.

32. Poretti A, Hausler M, von Moers A, Baumgartner B, Zerres K, Klein A, et al. Ataxia, intellectual disability, and ocular apraxia with cerebellar cysts: a new disease? Cerebellum. 2014;13(1):79-88.

33. Aldinger KA, Mosca SJ, Tetreault M, Dempsey JC, Ishak GE, Hartley T, et al. Mutations in LAMA1 cause cerebellar dysplasia and cysts with and without retinal dystrophy. Am J Hum Genet. 2014;95(2):227-34.

34. Bahi-Buisson N, Poirier K, Fourniol F, Saillour Y, Valence S, Lebrun N, et al The wide spectrum of tubulinopathies: what are the key features for the diagnosis? Brain. 2014;137(Pt 6):1676-700.

35. Poretti A, Huisman TA, Scheer I, Boltshauser E. Joubert syndrome and related disorders: spectrum of neuroimaging findings in 75 patients. AJNR Am J Neuroradiol. 2011;32(8):1459-63.

36. Nowak DA, Trost HA. Lhermitte-Duclos disease (dysplastic cerebellar gangliocytoma): a malformation, hamartoma or neoplasm? Acta Neurol Scand. 2002;105(3):137-45.

37. Poretti A, Meoded A, Huisman TA. Neuroimaging of pediatric posterior fossa tumors including review of the literature. J Magn Reson Imaging. 2012;35(1):32-47.

38. Toelle SP, Poretti A, Weber P, Seute T, Bromberg JE, Scheer I et al. Cerebellar Hypoplasia and Dysmorphia in Neurofibromatosis Type 1. Cerebellum. 2015;epub Mar 3.

\section{Submit your next manuscript to BioMed Central and take full advantage of:}

- Convenient online submission

- Thorough peer review

- No space constraints or color figure charges

- Immediate publication on acceptance

- Inclusion in PubMed, CAS, Scopus and Google Scholar

- Research which is freely available for redistribution

Submit your manuscript at www.biomedcentral.com/submit 